\title{
Antioxidative activity of the buckwheat polyphenol rutin in combination with ovalbumin
}

\author{
RIE AWATSUHARA ${ }^{1}$, KAZUKI HARADA $^{2}$, TOSHIMICHI MAEDA ${ }^{2}$, TAKAHIRO NOMURA ${ }^{1}$ and KEIKO NAGAO ${ }^{3}$ \\ ${ }^{1}$ Department of Food and Nutrition, Kanazawa Gakuin College, Ishikawa 920-1392; ${ }^{2}$ Graduate School of Fisheries Science, \\ National Fisheries University, Yamaguchi 759-6595; ${ }^{3}$ Department of Food and Nutrition, \\ Faculty of Home Economics, Tokyo Kasei University, Tokyo 173-8602, Japan
}

Received September 17, 2009; Accepted November 16, 2009

DOI: 10.3892/mmr_00000228

\begin{abstract}
Buckwheat flour is well known for its highly antioxidative ingredient, rutin. We have undertaken to examine alterations in the characteristics of rutin treated with various proteins. In this study, the radical scavenging activities of a rutin-ovalbumin complex were examined. Dissolved rutin hydrate and ovalbumin were combined and boiled in water for $10 \mathrm{~min}$. In the resulting rutin-ovalbumin complex, a new high molecular weight peak was detected using gel permeation chromatography analysis, and an existing high molecular weight area of ovalbumin was observed to be increased by the addition of rutin. This suggested that ovalbumin molecules produce a complex through their interaction with rutin. Alkaline luminol chemiluminescence and electron spin resonance analysis revealed the formation of a rutin-ovalbumin complex that markedly enhanced the peroxyl, but not the hydroxyl, radical scavenging activity of rutin. Rutin also demonstrated antioxidative activity against hydroxyl radicals in a DNA protection assay. We therefore conclude that, compared with ovalbumin or rutin alone, the rutin-ovalbumin complex has improved antioxidative activities in the form of enhanced peroxyl radical scavenging activity and DNA protection from apurinic/apyrimidinic site formation caused by hydroxyl radicals.
\end{abstract}

\section{Introduction}

Active oxygen species generated in the body cause damage to DNA and the lipid membrane structure of cells and play a role in the process of aging and the development of cancer (1). It is therefore important to control the amount of excess active oxygen in our body. Antioxidative components found in food have been shown to be effective as scavengers of active

Correspondence to: Dr Rie Awatsuhara, Department of Food and Nutrition, Kanazawa Gakuin College, 10 Sue-machi, Kanazawa-shi, Ishikawa 920-1392, Japan

E-mail: r-awa@kanazawa-gu.ac.jp

Key words: rutin, ovalbumin, DNA protection, chemiluminescence, electron spin resonance oxygen (2). We have been studying the antioxidative activity of rutin, a flavonoid of buckwheat. Buckwheat and/or tartary buckwheat flours are used to make 'Soba', a typical Japanese thin noodle. Adding egg whites to buckwheat flour during 'Soba' processing makes it easier to form the dough.

Research has indicated that rutin and quercetin may be metabolized by albumin-mediated transport in the body (3-5). Papadopoulou et al reported a binding affinity between flavonoids and bovine serum albumin (BSA), ranking in the order of quercetin $>$ rutin $>$ epicatechin $=$ catechin (4). This suggests the possibility of a chemical interaction between rutin and ovalbumin (OVA) during the boiling of 'Soba', which may influence the antioxidative activity of either substance. We previously reported that heating rutin with water-soluble whey protein isolate (WPI), a milk ingredient, results in the alteration of its antioxidative activity (6). Formation of a rutin-WPI complex enhanced the peroxyl and hydroxyl radical scavenging activities of rutin or WPI. We also studied the effects of the interation of another flavonoid, tannin, and WPI on the physical properties of gelatin gel (7), $\mathrm{pH}$ alteration on the gelation by rutin in gelatin solution (8) and the alteration of rheological properties of buckwheat noodles for different content ratios of rutin and wheat gluten (9).

In this study, the interaction between rutin and OVA heated together was first investigated using gel permeation chromatography (GPC). Next, chemiluminescence and electron spin resonance (ESR) were respectively used to determine whether or not this interaction enhanced the peroxyl or the hydroxyl radical scavenging activity of the combined substances in comparison with the activity of rutin or OVA alone. The antioxidative activity of rutin was then further examined using a new method that detects the level of DNA protection from the apurinic/apyrimidinic (AP) site formation caused by hydroxyl radicals. The results suggest the possibility of the formation of a rutin-OVA complex with improved antioxidative function.

\section{Materials and methods}

Chemicals. 2,2'-Azobis(2-amidinopropane)dihydrochloride (AAPH), sodium tetraborate decahydrate (borax), hydrochloric acid $(\mathrm{HCl})$ and $\beta$-carotene were obtained from Wako Pure Chemical, Ltd. (Osaka, Japan). Luminol and cytochrome $c$ from horse heart were obtained from Nacalai Tesque 
(Kyoto, Japan). Hydrogen peroxide $\left(\mathrm{H}_{2} \mathrm{O}_{2}\right)$ was from Santoku Chemical Co., Ltd. (Tokyo, Japan). Iron (II) sulfate heptahydrate $\left(\mathrm{FeSO}_{4} \cdot 7 \mathrm{H}_{2} \mathrm{O}\right)$ was from Sigma-Aldrich Japan (Tokyo, Japan). The spin trapping reagent 5,5-dimethyl-1-pyrroline $\mathrm{N}$-oxide (DMPO) was from Tokyo Kasei Kogyo Co., Ltd. (Tokyo, Japan). Tris (hydroxymethyl) aminomethane and deoxyribonucleic acid (DNA) sodium salt from salmon testes, rutin hydrate, ovalbumin (OVA; from chicken egg white, grade V) and astaxanthin were from Sigma-Aldrich (St. Louis, MO, USA). Ethylenediamine-N,N,N',N'-tetraacetic acid disodium salt dihydrate (EDTA-2Na) and a DNA damage quantification kit were from Dojindo Laboratories (Kumamoto, Japan). The kit contained an aldehyde reactive probe (ARP; N'-amino-oxymethylcarbonylhydrazino-D-biotin), horseradish peroxidase (HRP)-streptavidin, ARP-DNA standard, DNA binding buffer including TMBZ (3,3',5,5'-tetramethylbenzidine) and phosphate-buffered saline (PBST; with $0.05 \%$ Tween-20, pH 7.4).

Sample preparation. Rutin hydrate (100 ppm) and 3\% (w/v) OVA were combined in a flask in a total volume of $10 \mathrm{ml}$ and boiled in water for $10 \mathrm{~min}$. The sample was then quickly cooled with ice water. Rutin solution (100 ppm) and 3\% OVA solution as the control were separately but similarly prepared, and defined as the original solutions (100\%).

Chromatographic analysis. The HPLC system consisting of an L-6200 pump, an L-4200 UV detector (Hitachi HighTechnologies Co., Tokyo, Japan) and a Shodex Asahipak GS-620 column $(7.5 \times 30 \mathrm{~mm})$ with a Shodex Asahipak GS-1G short guard column (Showa Denko K.K., Tokyo, Japan) was used for the assay. The mobile phase was $100 \mathrm{mM}$ phosphate buffer ( $\mathrm{pH}$ 6.8) with a flow rate of $1.0 \mathrm{ml} / \mathrm{min}$, and the eluate was monitored by absorption at $220 \mathrm{~nm}$. The 10 -fold diluted samples were injected into the HPLC column after filtration with a membrane filter $(0.45 \mu \mathrm{m})$. Experimental data was analyzed by the Chromato Integrator D-2500 (Hitachi HighTechnologies Co.). The calibration curve was constructed by plotting the various molecular weight pullulans (Shodex Standard P-82; Showa Denko K.K.). A rutin concentration of $500 \mathrm{ppm}$ was used in the chromatographic experiment.

Chemiluminescence analysis. Chemiluminescence analysis was performed as previously described in detail (6). In brief, phosphate buffer was used to prepare a $40 \mathrm{mM}$ AAPH solution and to dilute the samples. The AAPH solution $(0.2 \mathrm{ml})$ was added to $0.2 \mathrm{ml}$ of each diluted sample, then the samples were incubated at $37^{\circ} \mathrm{C}$ for $2 \mathrm{~min}$. Immediately after incubation, $0.2 \mathrm{ml}$ luminol solution was added, and the chemiluminescence intensity was detected by a photon counter (Lumitester C-100; Kikkoman Co., Tokyo, Japan). One relative light unit (RLU) represents 43.48 photons/sec.

Electron spin resonance analysis. ESR was performed as previously described (6). In brief, hydroxyl radicals were generated by the DMPO-supplemented Fenton's reaction with or without examination of the samples (rutin, OVA and rutin-OVA complex). The addition of $8.8 \mathrm{mM} \mathrm{H}_{2} \mathrm{O}_{2}(50 \mu \mathrm{l})$ to the above reaction mixture $(320 \mu \mathrm{l})$ was used to initiate the Fenton's reaction, which occurs as in the chemical equation:
$\mathrm{Fe}^{2+}+\mathrm{H}_{2} \mathrm{O}_{2} \rightarrow \mathrm{Fe}^{3+}+\cdot \mathrm{OH}+\mathrm{OH}^{-}$. After 1 min of hydroxyl radical generation, spin adduct DMPO-OH• was measured using the ESR spectrometer (JES-FR30; JEOL Ltd., Tokyo, Japan). ESR measurement conditions were as follows: output, $4 \mathrm{~mW}$ (9.4 GHz); magnetic field, 342.790 $\pm 5 \mathrm{mT}$; modulation amplitude, $0.079 \mathrm{mT}$; time constant, $0.1 \mathrm{sec}$; sweeping time, $1 \mathrm{~min}$; and amplification ratio, 32-125.

Calculation of the $I C_{50}$ value of peroxyl or hydroxyl radical scavenging. $\mathrm{IC}_{50}$ values were defined as the concentrations of the individual rutin, OVA and rutin-OVA complex samples that reduced the control RLU value (chemiluminescence intensity generated by peroxyl radical) or the control peak height ratio of ESR (generation of hydroxyl radical) by half. The antioxidative value was calculated by the formula: (log Io/I) x 100, where Io is the RLU value or ESR peak height ratio of the control, and I is the RLU value or ESR peak height ratio of the samples. Thus, the $\mathrm{IC}_{50}$ values were the concentration of samples at Io/I $=1 / 2$, assessed using the antioxidative results of RLU or ESR obtained from the experiments (10).

Preparation of the protected DNA samples. Salmon testes DNA was prepared in TE buffer $(10 \mathrm{mM}$ Tris- $\mathrm{HCl}, 1 \mathrm{mM}$ EDTA-2Na, $\mathrm{pH}$ 7.5) at a concentration of $1 \mathrm{mg} / \mathrm{ml}$. Hydroxyl radical damaged or protected DNA samples were prepared according to a previously described method (11). In brief, $300 \mu \mathrm{l}$ of DNA solution was mixed with $50 \mu \mathrm{l}$ of $1.0 \mathrm{mM}$ $\mathrm{FeSO}_{4}$ together with or without $250 \mu \mathrm{l}$ of the antioxidant samples, and immediately incubated at $37^{\circ} \mathrm{C}$ for $5 \mathrm{~min}$. Fenton's reaction was initiated by adding $50 \mu \mathrm{l}$ of $8.8 \mathrm{mM}$ $\mathrm{H}_{2} \mathrm{O}_{2}$ to the mixture (negative control, $\mathrm{H}_{2} \mathrm{O}$ instead of $\mathrm{H}_{2} \mathrm{O}_{2}$ ). The mixture was incubated at $37^{\circ} \mathrm{C}$ for another $15 \mathrm{~min}$ and was cooled down on ice.

ARP labeling of DNA and determination of the number of $A P$ sites in DNA. DNA labeling with ARP (aldehyde reactive probe; N'-aminooxymethylcarbonylhydrazino-D-biotin) was as described previously $(12,13)$. In brief, $10 \mu \mathrm{l}$ of the aboveprepared DNA $(100 \mu \mathrm{g} / \mathrm{ml})$ was mixed with $10 \mu \mathrm{l}$ of ARP solution and biotinylated using the DNA damage quantification kit. For purification of the DNA samples, the Nanosep 30K device (molecular weight, 30,000; Pall Corp., NY, USA) was used. The ARP-labeled DNA was prepared in $400 \mu \mathrm{l}$ of TE buffer, then placed in the wells of a 96-well microplate (\#650061, Greiner Bio-One, Germany) and examined using the HRP-streptavidin and TMBZ provided in the kit. The absorbance at $650 \mathrm{~nm}$ was determined using a microplate reader (Model 680; Bio-Rad Laboratories, Tokyo, Japan). Dual samples were examined for each experiment. For calibration, $60 \mu \mathrm{l}$ each of standard solutions $(0,2.5,5,10,20$ and $40 \mathrm{AP}$ sites per $1 \times 10^{5} \mathrm{bp}$, respectively) were applied as a duplicate series in 96-well plates.

Calculation of the ratio of DNA protection. The ratio of DNA protection (\%) was defined as: $[(\mathrm{A}-\mathrm{B})-(\mathrm{C}-\mathrm{B})] \times 100 /(\mathrm{A}-\mathrm{B})=$ $(\mathrm{A}-\mathrm{C}) \times 100 /(\mathrm{A}-\mathrm{B})$, where $\mathrm{A}$ is the number of $\mathrm{AP}$ sites per $10^{5}$ bp after exposure to hydroxyl radicals in Fenton's reaction, $\mathrm{B}$ is the number of AP sites per $10^{5} \mathrm{bp}$ without exposure, and $\mathrm{C}$ is the number of AP sites per $10^{5} \mathrm{bp}$ after examination with antioxidants. 


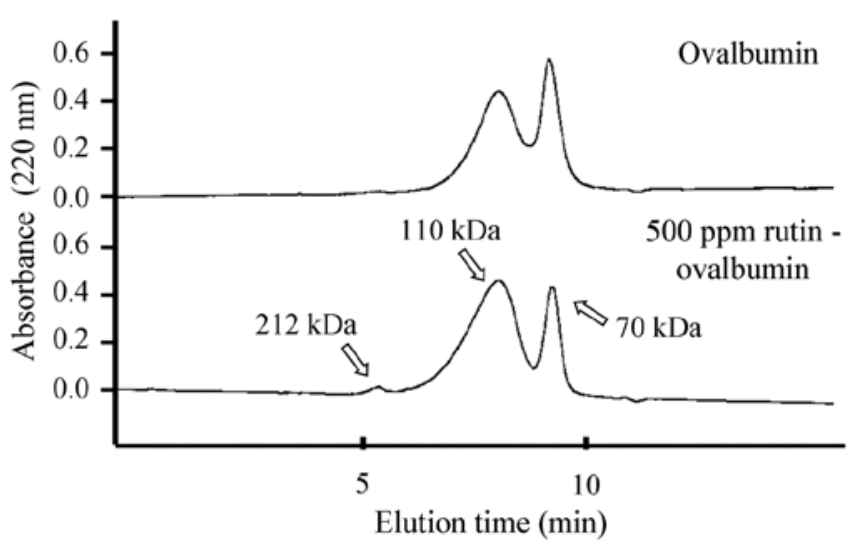

Figure 1. Molecular weight distribution in the OVA control (top) and in OVA with 500 ppm rutin (bottom) determined by gel permeation chromatography.

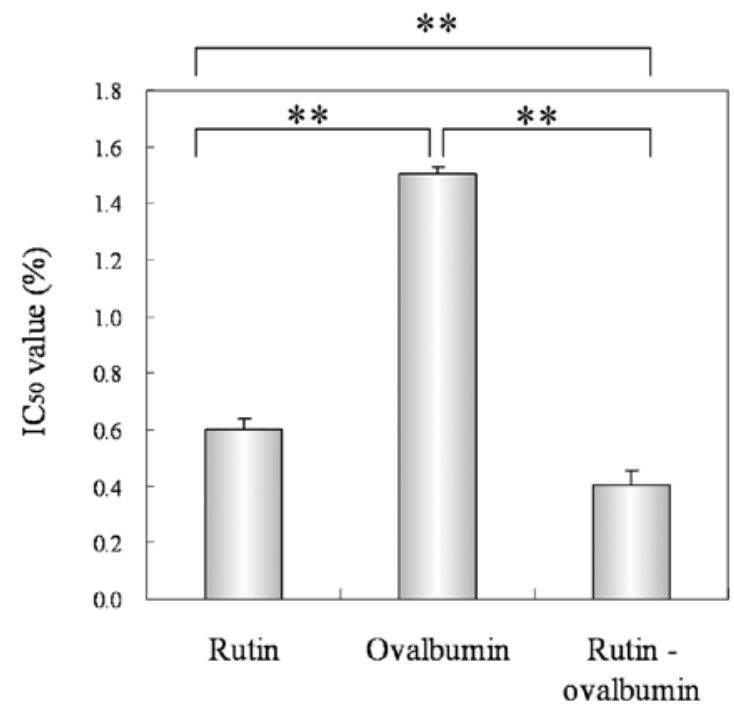

Figure 2. $\mathrm{IC}_{50}$ values (\%) of the peroxyl radical scavenging activities of rutin, OVA and the rutin-OVA complex measured using chemiluminescence. ${ }^{* *} \mathrm{p}<0.01 ; \mathrm{n}=3-8$.

\section{Results and Discussion}

In the results of gel permeation chromatography (GPC), OVA alone displayed two large peaks at 70 and $110 \mathrm{kDa}$ (Fig. 1; top). By contrast, the OVA sample boiled with rutin displayed a $212-\mathrm{kDa}$ peak as a new polymer (Fig. 1; bottom). The relative size of the $70-\mathrm{kDa}$ peak was not altered by the addition of rutin; however, the $110-\mathrm{kDa}$ peak became higher and broader towards the high molecular range of the chromatography compared to the OVA alone (Fig. 1; top). This observation suggests that the addition of rutin induced a shift in the molecular weight of OVA, from 70 or $110 \mathrm{kDa}$ to a larger one. We previously reported on the increase of the high molecular weight distribution of a WPI-gelatin complex by the addition of tannin to the solution (7). These results suggest that flavonoids may have the ability to promote an increase in the molecular weight of various proteins. Manach et al (3) found that the high affinity of quercetin (aglycon of rutin) to albumin may be caused by its structure, composed of numerous phenolic groups with hydrophobic interaction

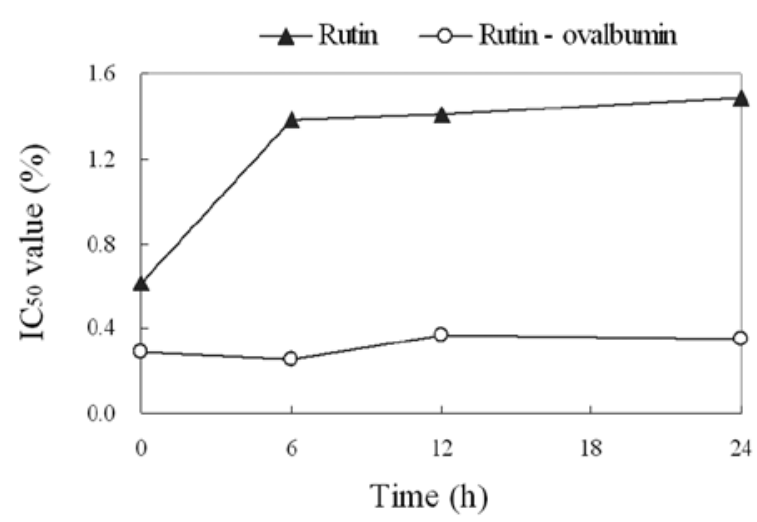

Figure 3. Changes in the peroxyl radical scavenging activity of rutin and the rutin-OVA complex at various intervals after sample preparation. $\mathbf{\Delta}, 100 \mathrm{ppm}$ rutin; O, OVA-rutin complex.

sites. They concluded that the phenolic groups of quercetin may provide a multiplicity of potential binding sites for the hydrogen bond. The authors also reported the bathochromic effect of albumin by the albumin/quercetin molar ratio, i.e., the absorption wavelength of human albumin shifted from 380 to $434 \mathrm{~nm}$ when the albumin/quercetin molar ratio reached 20. We found a similar absorption wavelength shift when rutin was treated with OVA (data not shown). These observations strongly suggest an intimate chemical interaction between OVA and rutin. Doronicheva et al demonstrated, using chemiluminescence, that the binding of flavonoids to the protein region of catalase contributed to an improvement in the activity of the enzyme (14). However, there are no reports on the alteration of the antioxidative activity of the rutin-OVA complex during the heating process. If the antioxidative activity of rutin is enhanced by interaction with OVA, the function of 'Soba' (a Japanese buckwheat noodle) may be improved by the addition of egg whites during the cooking process.

Fig. 2 shows the $\mathrm{IC}_{50}$ values of the peroxyl radical scavenging activities of $100 \mathrm{ppm}$ rutin, 3\% OVA and the rutin-OVA complex, respectively. The $\mathrm{IC}_{50}$ values are the final concentrations of the samples that reduced the intensity of chemiluminescence caused by the peroxyl radical by half (6). Thus, in this assay, low $\mathrm{IC}_{50}$ values indicated high antioxidative activity. The $\mathrm{IC}_{50}$ value of the rutin-OVA complex $(0.43 \pm 0.05 \%)$ was lower than that of rutin alone $(0.60 \pm 0.04 \%)$ or OVA alone $(1.51 \pm 0.02 \%)$. These results indicate that the rutin-OVA complex acquired additional antioxidative activity for peroxyl radical scavenging.

Buckwheat foods are usually consumed after a period of preservation (i.e., there is a period of several days between production and consumption). Thus, the time decay of the peroxyl radical scavenging activity of rutin and rutin-OVA was examined. The $\mathrm{IC}_{50}$ value of rutin was $0.61 \%$ at the start time, and markedly increased by up to $1.38 \%$ within $6 \mathrm{~h}$ (Fig. 3). After $24 \mathrm{~h}$, the $\mathrm{IC}_{50}$ value of rutin reached $1.49 \%$ due to the gradual decay of its scavenging activity. On the other hand, no alteration in the $\mathrm{IC}_{50}$ value of rutin-OVA was observed during a period of $24 \mathrm{~h}$; thus, the effect of time decay on the activity of the complex may be negligible. The $\mathrm{IC}_{50}$ value of OVA demonstrated a similar tendency. Thus, the depression of the antioxidative activity of rutin is suppressed by its interac- 


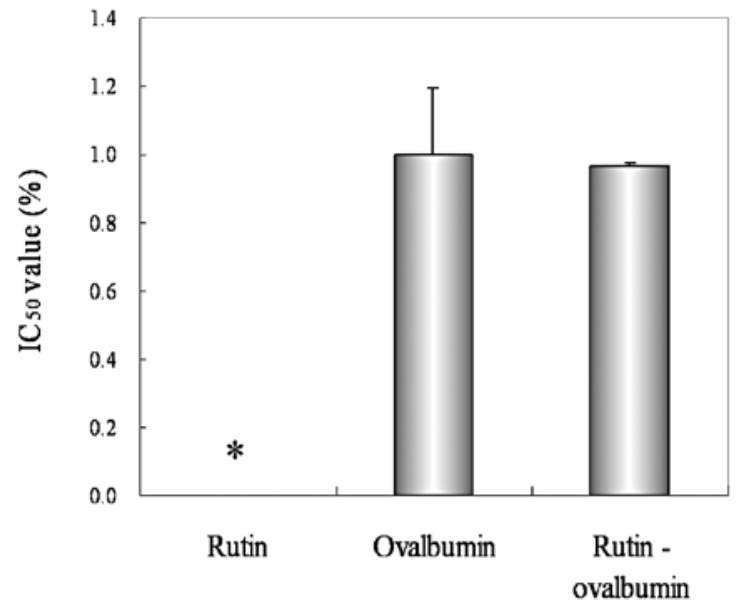

Figure $4 . \mathrm{IC}_{50}$ values (\%) of the hydroxyl radical scavenging activities of rutin, OVA and the rutin-OVA complex measured using electron spin resonance. ${ }^{*} \mathrm{IC}_{50}$ value of rutin for hydroxyl radical scavenging activity $>100 \%$; $\mathrm{n}=3-8$.

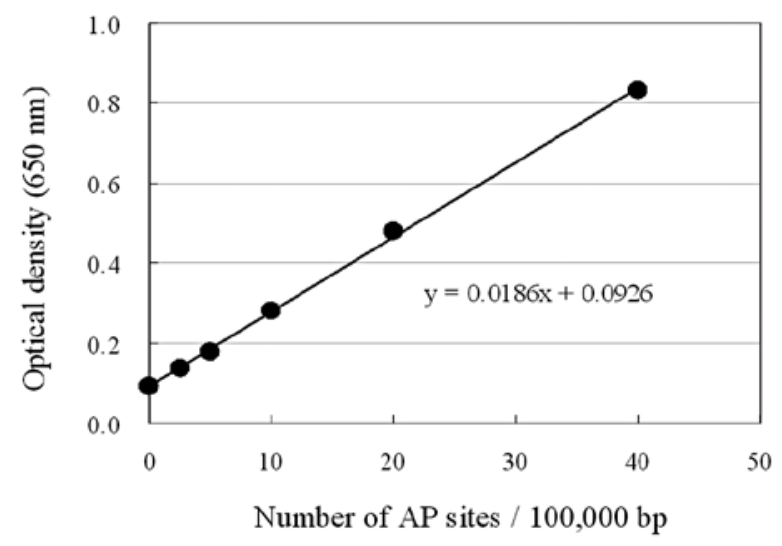

Figure 5. Calibration curve using the ARP-DNA standard. Vertical and horizontal axes indicate the optical density at $650 \mathrm{~nm}$ and the number of AP sites per $10^{5} \mathrm{bp}$, respectively.

tion with OVA. This suggests that the antioxidative activity of rutin is controllable, and that the stability of the functional improvement may be maintained by the addition of ovalbumin (or egg whites) to buckwheat foods.

The $\mathrm{IC}_{50}$ values of the hydroxyl radical scavenging activities of $100 \mathrm{ppm}$ rutin, $3 \%$ OVA and the rutin-OVA complex are shown in Fig. 4. For the hydroxyl radical scavenging, the $\mathrm{IC}_{50}$ value of $100 \mathrm{ppm}$ rutin was $>100 \%$, indicating no effect at this concentration. The $\mathrm{IC}_{50}$ values of OVA and the rutin-OVA complex were $1.00 \pm 0.20$ and $0.97 \pm 0.01 \%$, respectively. We previously demonstrated that a similar combination of rutin with WPI had synergistic effects on hydroxyl radical scavenging activity, leading the rutin-WPI complex to have accelerated radical-reducing activity (6). In contrast, the hydroxyl radical scavenging activities of the rutin-OVA complex improved very slightly compared to those of the OVA alone. The hydroxyl radical scavenging activity of rutin and vitamin $B_{2}$ was also found to be low upon ESR analysis with CYPMPO (5-(2,2-dimethyl1,3-propoxy cyclophosphoryl)-5-methyl-1-pyrroline-N-oxide) (15). The hydroxyl radical scavenging activity of the rutinprotein complex may be influenced by the activity of the protein itself or by the protein species combined.

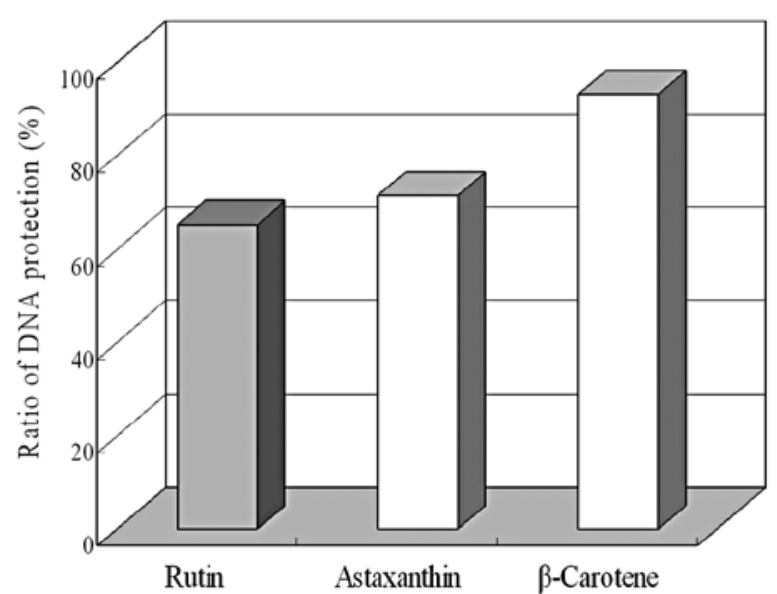

Figure 6. Ratio of DNA protection (\%) against hydroxyl radical damage in the Fenton's reaction solution.

Chen et al reported that rutin and quercetin from Ginkgo protected cerebellar granule cells from apoptosis caused by oxidative damage induced by hydroxyl radicals (16). We also studied the protection of DNA from the damage induced by hydroxyl radicals. AP sites are the major types of damage generated by hydroxyl radicals in double-stranded DNA. The biotinylated reagent ARP specifically reacts with the aldehyde of AP sites; thus, HRP-conjugated streptavidin can detect AP sites by the colorimetric reaction (13). This method has been adopted to detect the antioxidative activity of food or food-derived materials $(11,17)$. The number of AP sites on DNA damaged by hydroxyl radicals was obtained from the calibration curve using AP site standard DNA (Fig. 5). The estimated DNA protection ratio of rutin was compared with that of astaxanthin and $\beta$-carotene (Fig. 6). Rutin (65.1\%) showed a similar level of DNA protection from damage by the hydroxyl radical as astaxanthin (71.2\%), but was greatly inferior to carotene (93.1\%), used as a positive control. We also determined the preliminary protection result of the rutin-OVA complex to be approximately $80 \%$ (data not shown).

These results indicate the high antioxidative activity of rutin, not only against peroxyl radicals, but also against hydroxyl radicals, using different detection methods. This antioxidative activity was improved by the chemical interaction of rutin with various proteins; in particular, the rutin-OVA complex may have improved DNA protection activity. The heating of rutin with OVA promoted complex formation, suggesting the possibility of the functional improvement of rutin during food processing, such as noodle making. Further research is necessary to determine possible improvements to the function of 'Soba'.

\section{Acknowledgements}

The authors are grateful to Ms. Tomoka Fujii, Ms. Nanae Higashi (Department of Food and Nutrition, Kanazawa Gakuin College, Japan) and Mr. Yoshiro Hasegawa (Graduate School, National Fisheries University, Japan) for their technical assistance. This study was supported, in part, by research grants from the Iijima Memorial Foundation for the Promotion of Food Science and Technology in 2007, and from Nikkoku Seifun Co. Ltd. 


\section{References}

1. Kehrer JP: Free radicals as mediators of tissue injury and disease. Crit Rev Toxicol 23: 21-48, 1993.

2. Havsteen BH: The biochemistry and medical significance of the flavonoids. Pharmacol Ther 96: 67-202, 2002.

3. Manach C, Morand C, Texier O, Favier M-L, Agullo G, Demigné C, Régérat $\mathrm{F}$ and Rémésy $\mathrm{C}$ : Quercetin metabolites in plasma of rats fed diets containing rutin or quercetin. J Nutr 125: 1911-1922, 1995.

4. Papadopoulou A, Green RJ and Frazier RA: Interaction of flavonoids with bovine serum albumin: a fluorescence quenching study. J Agric Food Chem 53: 158-163, 2005.

5. Lu QH, Ba CD and Chen DY: Investigating noncovalent interactions of rutin-serum albumin by capillary electrophoresis-frontal analysis. J Pharm Biomed Anal 47: 888-891, 2008.

6. Awatsuhara R, Harada K, Nomura T, Nikaido O and Nagao K: Alteration of antioxidative activity of rutin treated with food protein. J Clin Biochem Nutr 43: S512-S515, 2008.

7. Awatsuhara R and Nagao K: Effects of tannin addition on physical properties of whey protein-containing gelatin gels. J Integr Stud Diet Habits (in Japanese) 19: 28-32, 2008.

8. Awatsuhara R, Nomura T, Nikaido O and Nagao K: Effect of $\mathrm{pH}$ value on the gelation of a rutin-containing gelatin solution and on the crystallization of rutin. J Cookery Sci Jpn (in Japanese) 40 : 166-172, 2007.

9. Awatsuhara R, Higuchi F, Tsuchida K and Nagao K: Effects of rutin and wheat gluten contents on the rheological properties of buckwheat noodles. J Cookery Sci Jpn (in Japanese) 41: 319-326, 2008.

10. Nagatsuka N, Harada K, Ando M and Nagao K: Effect of soy sauce on the antioxidative capacity of the gelatin gel food 'Nikogori' measured using the chemiluminescence method. Int J Mol Med 16: 427-430, 2005.
11. Harada K, Makino Y, Yamauchi T, Fukuda N, Tamaru M, Okubo Y, Maeda T, Fukuda Y and Shiba T: Efficacy of puffer fish (Takifugu rubripes) sauce in reducing hydroxyl radical to DNA assessed using the apurinic/apyrimidinic site method. Int J Mol Med 20: 309-314, 2007.

12. Kubo K, Ide H, Wallace SS and Kow YW: A novel, sensitive, and specific assay for abasic sites, the most commonly produced DNA lesion. Biochemistry 31: 3703-3708, 1992.

13. Ide H, Akamatsu K, Kimura Y, Michiue K, Makino K, Asaeda A, Takamori Y and Kubo K: Synthesis and damage specificity of a novel probe for the detection of abasic sites in DNA. Biochemistry 32: 8276-8283, 1993.

14. Doronicheva N, Yasui H and Sakurai H: Chemical structuredependent differential effects of flavonoids on the catalase activity as evaluated by a chemiluminescent method. Biol Pharm Bull 30: 213-217, 2007.

15. Endo N: Evaluation of anti-oxidation activity of food by spintrapping ESR measurements. Annual Report of The Wakasa Wan Energy Research Center (in Japanese) 9: 41-44, 2006.

16. Chen C, Wei T, Gao Z, Zhao B, Hou J, Xu H, Xin W and Packer L: Different effects of the constituents of EGb761 on apoptosis in rat cerebellar granule cells induced by hydroxyl radicals. Biochem Mol Biol Int 47: 397-405, 1999.

17. Makino Y, Fujisawa H, Okazaki K and Hirata T: Depression effect of foods against DNA damage caused by hydroxyl radical evaluated by biotinylation of apurinic/apyrimidinic sites. Annual Report of Kagawa Prefectural Industrial Technology Center (in Japanese) 2: 133-136, 2001. 
